\title{
L'HYBRIDATION DANS LES POPULATIONS NATURELLES DE SALMONIDÉS DANS LE SUD-OUEST DE L'EUROPE ET EN MILIEU EXPÉRIMENTAL.
}

\author{
E. BEALL (1), P. MORAN (2), A. PENDAS (2), J. IZQUIERDO (2), E. GARCIA VAZQUEZ (2),
}

avec la collaboration technique de S. GLISE (1), J.C. VIGNES (1) et L. BARRIERE (1).

(1) Ecologie des Poissons, INRA Station d'Hydrobiologie, B.P. 3, 64310 Saint-Pée-sur-Nivelle, France.

(2) Departamento de Biologia Funcional, Area de Genetica, Facultad de Medicina, Universidad de Oviedo, 33006 Oviedo, Espagne.

\section{RÉSUMÉ}

L'hybridation interspécifique entre le saumon atlantique et la truite commune dans la nature a été mise en évidence dans différents pays d'Europe et au Canada. Une étude a été entreprise pour examiner son incidence dans des populations de salmonidés de certaines rivières des Asturies (nord de l'Espagne) et du sud-ouest de la France. Elle a été complétée par des expériences en milieu contrôlé pour déterminer les causes et les conditions de la disparition des barrières comportementales permettant le maintien de l'isolement reproducteur. Les hypothèses de travail étaient que l'hybridation pouvait être favorisée par le comportement de " sneaker " des tacons mâles précoces et par les repeuplements en juvéniles des deux espèces. Les résultats obtenus confirment que l'hybridation entre la truite et le saumon est un phénomène répandu, qui peut affecter localement des fractions significatives des populations $(9,4 \%$ sur la rivière Narcea dans les Asturies). Dans l'aire originelle de distribution des deux espèces, le croisement s'effectue dans le sens femelle saumon $x$ mâle truite. Dans les conditions normales de sympatrie, les barrières d'isolement reproducteur pré-appariement paraissent solides en raison du comportement agressif du mâle conspécifique dominant qui parvient à écarter efficacement les mâles compétiteurs hétérospécifiques. En l'absence de mâle conspécifique, l'hybridation peut avoir lieu. Cependant, les femelles modifient leur comportement, ralentissent leur activité de frai et le succès reproducteur, particulièrement chez la truite, diminue. Par ailleurs, les hypothèses de travail ne sont pas vérifiées. Pour éviter l'hybridation, il est recommandé au gestionnaire de veiller à la qualité et à la quantité des zones de reproduction, de maintenir l'équilibre des populations de géniteurs et de limiter les repeuplements en sujets non autochtones.

\section{HYBRIDIZATION IN NATURAL POPULATIONS OF SALMONIDS IN SOUTH-WEST EUROPE AND IN AN EXPERIMENTAL CHANNEL.}

\begin{abstract}
Interspecific hybridization between Atlantic salmon and brown trout has been found in different European countries and in Canada. A study was initiated to determine the incidence of hybrids in salmonid populations of some rivers in Asturias (North Spain) and Southwestern France. It was complemented by experiments in a controlled environment to examine the
\end{abstract}


conditions of the breaking down of reproductive isolation. Working hypotheses were that hybridization was favoured by the sneaking behaviour of precocious male salmon parrs and the stocking of juveniles of the two species. Results confirmed the widespread occurrence of this phenomenon. Significant proportions of salmonid populations were locally affected by hybridization ( $9.4 \%$ in the Narcea river, Asturias). Within the native range of the two species, the direction of crossing was female salmon $x$ male trout. Under normal sympatric conditions, pre-mating behavioural isolating barriers seemed to be strong because of the aggressive behaviour of the dominant conspecific males who successfully chased away competing heterospecific males. In the absence of conspecific males hybridization did occur. However, females modified their spawning behaviour and their reproductive success decreased, particularly in trout. Working hypotheses were not confirmed. To avoid hybridization, managers should make sure that sufficient numbers of spawning zones are available, that spawner populations are well balanced, and that the stocking of exotic juveniles is limited.

\section{1 - INTRODUCTION}

L'hybridation entre espèces de salmonidés, et plus particulièrement entre le saumon atlantique, Salmo salar L., et la truite commune, S. trutta L., est un phénomène connu depuis longtemps car pratiqué en pisciculture depuis le 19ème siècle à des fins d'élevage. Certains hybrides sont fertiles et peuvent donner des descendants F2 ou rétrocroisés (CHEVASSUS, 1979). L'hybridation naturelle, intra et interspécifique, pose de nombreux problèmes aux gestionnaires, dont la tâche est de protéger les populations. En effet, si les descendants sont fertiles, le processus d'hybridation introgressive peut causer la perte génétique d'une espèce, sous-espèce ou population unique (CAMPTON, 1987). S'ils ne sont pas fertiles, cette hybridation conduit à un détournement d'une partie du potentiel reproducteur des espèces considérées qui n'est pas sans conséquences sur la gestion de ces ressources. Grâce aux techniques électrophorétiques modernes, l'identification d'hybrides est maintenant possible sans ambiguité (NYMAN, 1970 ; GUYOMARD, 1978) et des études conduites sur des populations naturelles de saumon atlantique et/ou de truite commune ont démontré la présence d'hybrides dans de nombreux hydrosystèmes, avec des fréquences très variables.

Dans les îles britanniques, les premières études portant sur des effectifs importants de saumons adultes capturés dans les pêcheries commerciales (PAYNE et al., 1972) indiquent un faible taux d'hybrides, de l'ordre de 0,4\%. Une valeur comparable est trouvée par CROZIER (1984) sur les populations de truites juvéniles et adultes du Lough Neagh en Irlande du nord. Des études plus récentes, portant sur des populations d'alevins de saumon en Ecosse, donnent un taux d'hybridation de l'ordre de $1 \%$ (YOUNGSON et al., 1993), chiffre comparable aux $1,2 \%$ cités par HURREL et PRICE (1991) pour des juvéniles de saumon dans le sud-ouest de l'Angleterre. Ces derniers auteurs trouvent également un taux de $2 \%$ pour les juvéniles de truite. Dans les pays scandinaves, une vaste étude électrophorétique n'a mis en évidence qu'un seul hybride sur 1500 saumons analysés (STAHL, in VERSPOOR, 1988). En Norvège, HEGGBERGET et al. (1988) ne trouvent aucun hybride sur un échantillon de plus de 1000 oeufs et alevins de saumons et de truites extraits de frayères dans quatre rivières. Par contre, des valeurs très élevées, $13 \%$ en moyenne, sont trouvées sur la rivière Grönan en Suède (JANSSON et al., 1991). SEMYONOVA et SLYN'KO (1988) indiquent des proportions d'hybrides variant de 0 à $0,5 \%$ sur des rivières de la péninsule de Kola (Russie), mais trouvent jusqu'à $31,4 \%$ sur certains affluents de la Mer Baltique. GARCIA DE LEANIZ et VERSPOOR (1989) déterminent des taux de 2,3\% sur quatre rivières de la côte cantabrique (Espagne). Au Canada, différents auteurs trouvent des taux d'hybridation variant de 0,9 à $4,7 \%$, entre le saumon atlantique autochtone et la truite commune introduite (BELAND et al., 1981 ; VERSPOOR, 1988 ; MCGOWAN et DAVIDSON, 1992 a).

La grande variabilité des résultats, pour un même système hydrographique ou une même région et d'une étude à l'autre, rend difficile l'interprétation du phénomène dans les populations naturelles. Une partie de cette variabilité peut être due à la technique d'échantillonnage, comme par exemple la prise en compte d'une seule des deux espèces. Une autre cause de sous- 
estimation pourrait résider dans l'échantillonnage des poissons aux stades adulte ou subadulte seulement. S'il existe des mortalités différentielles entre les hybrides et l'une ou l'autre des deux espèces, l'étude des poissons plus âgés ne donnera qu'une idée biaisée de la situation (HURREL et PRICE, 1991). Cette hypothèse semble soutenue par les données de VERSPOOR (1988), qui trouve $0 \%$ d'hybrides sur un échantillon de 198 saumons adultes, 0,3\% sur 330 smolts et $1,8 \%$ sur 394 juvéniles.

Différentes raisons peuvent être invoquées pour expliquer le phénomène de l'hybridation. Les deux espèces sont proches morphologiquement, et par la similarité des protéines et la phylogénie (JOHNSON et WRIGHT, 1986 ; STEARLEY, 1990). Leurs zones de reproduction sont semblables (HEGGBERGET et al., 1988), de même que leurs comportements sexuels. D'après CAMPTON (1987), l'hybridation naturelle serait plus fréquente chez les poissons que chez d'autres vertébrés en raison de la fécondation externe et de mécanismes comportementaux d'isolement faibles. II considère également qu'une abondance inégale des deux espèces parentales, la compétition pour un habitat de reproduction limité, et la susceptibilité de contacts secondaires entre formes ayant évolué récemment, peuvent intervenir. La maturation sexuelle précoce des tacons mâles et leur comportement de "sneaker " pourraient également favoriser l'hybridation entre la truite et le saumon (CROZIER, 1984 ; MCGOWAN et DAVIDSON, 1992 a). Par ailleurs, l'action de l'homme, par les modifications de l'habitat, les repeuplements, transferts de populations et introductions, est considérée comme pouvant augmenter l'incidence de l'hybridation (CAMPTON, 1987). Ainsi, la réduction des zones de frayères qui se chevauchent est considérée par JANSSON et al. (1991) comme une raison essentielle des taux élevés trouvés en Suède. Pour GARCIA DE LEANIZ et VERSPOOR (1989), c'est la structure déséquilibrée des populations, due à un manque de géniteurs d'un sexe de l'une ou l'autre espèce certaines années, alliée à des repeuplements massifs en juvéniles d'origine exogène, qui expliqueraient les données espagnoles. De même, les échappements, volontaires ou non, des élevages pourraient contribuer à augmenter l'hybridation (CROZIER, 1993 ; YOUNGSON et al., 1993), l'introgression ou le remplacement de populations sauvages (HINDAR et al., 1991). Enfin, l'introduction d'une espèce hors de son aire géographique de répartition, comme dans le cas de la truite commune au Canada, a favorisé l'hybridation avec l'espèce proche. L'ADN mitochondrial, transmis uniquement par la mère, a été analysé par MCGOWAN et DAVIDSON (1992 a) qui ont trouvé que le sens du croisement était femelle truite $x$ mâle saumon. Ils suggèrent que le biais dans la direction de l'hybridation est dû à la participation des tacons mâles précoces. Mais cette hypothèse est contredite par YOUNGSON et al. (1992, 1993) qui trouvent le croisement réciproque sur des hybrides adultes et juvéniles en Ecosse.

Au vu de ces résultats très variables et parfois contradictoires, il était nécessaire de faire le point sur la situation dans le sud-ouest de l'Europe, et plus particulièrement en France, pour laquelle il n'existe aucune donnée. Les rivières des Asturies dans le nord de l'Espagne et celles du nord du Pays Basque en France possèdent des populations de saumon et de truite relativement importantes, et certaines sont soumises à des opérations intensives de repeuplement en juvéniles d'origine étrangère. En outre, les saumons mâles juvéniles (tacons) ont tendance à maturer précocement en proportions plus importantes qu'ailleurs (NICIEZA et BRANA, 1995). Ainsi, sur la Nivelle, entre 50 et $100 \%$ d'une cohorte de mâles peuvent maturer dès l'âge de 0+ (HELAND et DUMAS, 1994).

L'équipe d'Ecologie des Poissons de I'INRA, à St-Pée-sur-Nivelle, dispose d'un ensemble d'installations permettant l'ètude expérimentale de la reproduction des salmonidés. Une collaboration entre l'équipe de génétique de l'Université d'Oviedo (Dr. E. GARCIAVAZQUEZ et coll.) et l'équipe d'Ecologie des Poissons de St-Pée a donc été établie, avec les objectifs suivants : 
1 - examiner l'incidence de l'hybridation entre le saumon et la truite dans differentes rivières du Pays Basque et des Asturies, en échantillonnant les juvéniles des deux espèces ;

2 - déterminer le sens des croisements par l'analyse de l'ADN mitochondrial ;

3 - évaluer l'impact des repeuplements;

4 - examiner expérimentalement dans quelles conditions s'abaissent les barrières d'isolement reproducteur ;

5 - déterminer le rôle des tacons mâles précoces.

\section{2 - MATÉRIEL ET MÉTHODES}

\section{1 - Echantillonnage en milieu naturel}

Pour évaluer l'incidence de l'hybridation dans les conditions naturelles, des prélèvements par pêche électrique ont été effectués sur une ou plusieurs stations de quatre rivières des Asturies (Narcea, Esva, Porcia et Cares) et deux rivières du Pays Basque (Nive et Nivelle). Les échantillons de juvéniles sont anesthésiés et le phénotype (truite ou saumon) est déterminé visuellement sur la base de l'apparence de la robe, la présence de marques de parr, l'échancrure de la nageoire caudale, la couleur de la nageoire adipeuse... Ils sont ensuite sacrifiés après mesure et pesée ; des morceaux de tissus et de nageoires sont prélevés, congelés et stockés à $-20^{\circ} \mathrm{C}$ au laboratoire d'Oviedo jusqu'à l'analyse. L'échantillon de la Nive est envoyé à l'Université de Cork (Irlande). En outre, un échantillon d'adultes de saumon, provenant des captures à la ligne sur la rivière Eo (Asturies), est inclus dans l'analyse des protéines par électrophorèse.

\section{2 - Expérimentation en milieu contrôlé}

Les possibilités d'hybridation ont été examinées expérimentalement dans le chenal de frai du Lapitxuri, station de terrain dépendant de l'INRA de St-Pée-sur-Nivelle. Différentes situations d'espèces, de sexe et de densité sont mises en place pour déterminer d'une part, le taux d'hybridation, la survie et autres caractéristiques des hybrides, et d'autre part, dans quelles circonstances disparaissent les barrières comportementales maintenant l'isolement reproducteur. Le chenal est une rivière artificielle de $130 \mathrm{~m}$ de long sur 2,8 $\mathrm{m}$ de large, divisée en 13 biefs de 23 à $28 \mathrm{~m}^{2}$, dans lesquels les conditions environnementales (vitesse du courant, débit, profondeur, abris) peuvent être manipulées. Plus de détails sur les installations et les caractéristiques morphologiques du site sont donnés dans BEALL et MARTY (1983).

La Nivelle possède une souche autochtone de saumon atlantique, constituée en majorité de castillons. Les géniteurs anadromes sont contrôlés lors de la remontée dans des pièges de capture situés sur les échelles à migrateurs équipant les barrages d'Uxondoa et d'Olha. Les tacons mâles précoces sont capturés à l'électricité lors des pêches d'inventaire effectuées dans la Nivelle au mois d'octobre. Des géniteurs de truite sauvage sont également capturés à l'électricité dans la Nivelle et ses affluents, peu avant la reproduction. Tous ces poissons sont gardés dans les installations de stabulation du Lapitxuri, jusqu'au moment de la reproduction en décembre. Des prélèvements de tissus ou de nageoires sont effectués selon les besoins.

Différents dèmes inter et intraspécifiques sont constitués dans des biefs séparés du chenal de frai (Tabl. II), début décembre, en 1992 et 1993 en fonction des poissons disponibles. Quatorze situations, où les géniteurs peuvent se reproduire naturellement et interagir, ont été examinées. Huit expériences ont été effectuées avec une femelle saumon castillon par bief, et six expériences avec cinq femelles truites par bief. Un nombre plus élevé de truites a été utilisé, en raison de leur taille beaucoup plus petite ( 30 à $430 \mathrm{~g}$ au lieu de 1,9 à $2,6 \mathrm{~kg}$ pour les saumons), afin d'obtenir des effectifs de descendants suffisants pour les analyses. Les femelles sont accompagnées de différentes combinaisons de mâles, de l'une et/ou de l'autre espèce. Dans chaque situation expérimentale, le début et la fin des activités de frai sont notés et des observations sur la fréquence de différents comportements considérés comme typiques de 
l'activité de frai (BEALL, 1994) sont effectuées visuellement ou grâce à une caméra vidéo de surveillance. En raison de difficultés techniques survenues au moment de la reproduction des truites en allopatrie, des observations complémentaires sur le comportement de reproduction de la truite sédentaire sauvage ont été obtenues sur un petit ruisseau de la réserve biologique de Muniellos, dans les Asturies.

Les données comportementales (moyennes de fréquence par périodes de $5 \mathrm{~min}$.) ont été analysées par des tests de KRUSKAL-WALLIS, équivalents non paramétriques de l'analyse de variance à une voie. Les résultats sont rapportés en détail dans BEALL (1996).

L'emplacement des frayères et des nids est noté avec précision pour suivre leur évolution spatio-temporelle. Une frayère est une zone homogène de substrat modifiée par la femelle, dans laquelle elle dépose ses oeufs dans une succession de nids ou poches (BEALL, 1994). Elle peut ainsi construire une à trois frayères (BEALL et MARTY, 1983). Après le frai, les femelles sont retirées et la rétention, c'est-à-dire le nombre d'ovules restant dans la cavité abdominale, est estimée par pression des flancs sur les poissons vivants ou par dissection des poissons morts. La fécondité moyenne du saumon atlantique de la Nivelle est de 1817 oeufs $/ \mathrm{kg}$ de femelle (BEALL et al., 1991), et celle de la truite a été estimée à partir de la relation : $F=1,35$ Poids $(\mathrm{g})$ +20 .

Le succès reproducteur de chaque femelle (ou nombre d'oeufs déposés) est estimé par la différence entre la fécondité calculée et la rétention observée.

A l'émergence des graviers, les alevins survivants sont capturés dans des filets placés en aval de chaque bief, et par pêche électrique par la méthode des captures successives. La survie peut ainsi être évaluée pour chaque situation, à partir de l'estimation du nombre d'oeufs déposés. Les nageoires adipeuses sont prélevées sur des échantillons de 50 à 100 juvéniles dans chaque bief et envoyées à Oviedo pour les analyses génétiques.

\section{3 - Analyses génétiques}

Toutes les analyses génétiques ont été effectuées à l'Université d'Oviedo, sauf celles concernant l'échantillon de la Nive. Les hybrides sont identifiés par électrophorèse de protéines, plus particulièrement les isozymes phosphoglucose isomérase et phosphoglucomutase, codées respectivement par les loci du muscle GPI-1,2* et PGM-2*. La méthode électrophorétique et l'interprétation des bandes de protéines suivent la procédure de VERSPOOR (1988). Une fois les hybrides identifiés, l'espèce maternelle est déterminée par analyse des patrons de restriction du mtADN, d'après les procédures de TAGGART et al. (1992) et ZIMMERMAN et al. (1988). Comme les patrons de restriction sont différents pour chaque espèce, il est possible de déterminer l'espèce maternelle pour chaque hybride.

L'identification des truites sauvages ou de repeuplement est basée sur le locus LDH-5*, considéré comme un marqueur génétique puissant distinguant les populations autochtones et exotiques de truites du sud de l'Europe (KRIEG et GUYOMARD, 1985 ; MORAN et al., 1991).

Les données de fréquence des hybrides ont été analysées par le test de $G$ d'indépendance (SOKAL et ROHLF, 1969).

\section{3 - RÉSULTATS}

\section{1 - Hybridation en milieu naturel}

Seize hybrides sur 719 poissons analysés, soit 2,2 \%, ont été détectés dans cinq rivières des Asturies et deux rivières du Pays Basque (Tabl. I). Les taux varient de $1 \%$ sur la Nivelle à $9,4 \%$ sur un échantillon de la Narcea. Une comparaison entre régions : $2,8 \%$ dans les Asturies et $1,2 \%$ en Pays Basque, indique que les taux sont homogènes (test de $G=1,08$ n.s.). Si l'on considère la répartition par phénotype, trois des hybrides ressemblent à des saumons et 13 à des truites (différence non significative, test de G). Sur 10 hybrides analysés pour l'origine maternelle par l'ADN mitochondrial, tous sont issus d'une femelle saumon. 
En ce qui concerne l'impact des truites de repeuplement, it peut être considéré comme très faible ou nul car tous les hybrides étaient du type $L D H-5^{\star} 100 / 100$. La participation de truites mâles de repeuplement dans les croisements peut être exclue, car ceux-ci devraient avoir le génotype $L D H-5^{*} 90 / 90$. Par contre, il n'a pas été possible de déterminer l'origine des femelles saumons, car il n'y a pas de marqueur absolu permettant de différencier les populations autochtones du sud des populations septentrionales qui ont généralement servi de source pour les approvisionnements en oeufs des piscicultures de repeuplement.

\section{Tableau I}

Fréquences de l'hybridation dans des rivières des Asturies et du Pays Basque et identification de la mère par l'ADNmt.

\section{Table I}

Occurrence of hybrids in rivers of Asturias and the Basque Country and identification of maternal origin according to mtDNA.

\begin{tabular}{|c|c|c|c|c|c|c|}
\hline \multirow[b]{2}{*}{ Rivière } & \multirow[b]{2}{*}{ Saumon } & \multirow[b]{2}{*}{ Truite } & \multicolumn{4}{|c|}{ Hybrides } \\
\hline & & & $\begin{array}{l}\text { phénotype } \\
\text { Saumon }\end{array}$ & $\begin{array}{l}\text { phénotype } \\
\text { Truite }\end{array}$ & $\%$ & $\begin{array}{l}\text { Femelle } \\
\text { parent }\end{array}$ \\
\hline Eo & $31^{*}$ & 0 & 1 & 0 & 3,2 & - \\
\hline Narceą 1 & 70 & 34 & 1 & 0 & 1,0 & - \\
\hline Narcea 2 & 0 & 33 & 0 & 1 & 3,3 & - \\
\hline Narcea 3 & 0 & 25 & 0 & 1 & 4,0 & - \\
\hline Narcea 4 & 0 & 32 & 0 & 3 & 9,4 & saumon \\
\hline Esva 1 & 24 & 46 & 0 & 1 & 1,4 & - \\
\hline Esva 2 & 0 & 36 & 0 & 2 & 5,5 & saumon \\
\hline Esva 3 & 0 & 30 & 0 & 1 & 3,3 & saumon \\
\hline Porcia & 23 & 9 & 0 & 1 & 3,1 & saumon \\
\hline Cares & 30 & 40 & 0 & 1 & 1,4 & saumon \\
\hline Nive & 32 & 0 & 1 & 0 & 3,1 & - \\
\hline Nivelle & 100 & 124 & 0 & 2 & 0,9 & saumon \\
\hline
\end{tabular}

* adultes 


\section{2 - Expériences en chenal de frai}

\subsubsection{Conditions de l'hybridation}

\section{Tableau II}

Conditions expérimentales, estimation du succès reproducteur, survie jusqu'à l'émergence et résultats des croisements impliquant soit une femelle saumon (exp. 1-8), soit 5 femelles truite (exp. 9-14) dans le chenal du Lapitxuri. $S=$ saumon ; $T$ = truite ; tac = tacon. Rétention : nombre total d'ovules restant dans la cavité abdominale des femelles après le frai.

Table II

Experimental conditions, reproductive success, survival to emergence, and results of crosses with either one female salmon (exp. 1-8), or 5 female trouts (exp. 9-14) in the Lapitxuri spawning channel. $S=$ salmon; $T=$ trout $;$ tac = precocious male parr. Retention : total number of ova left in the abdominal cavity of females after spawning.

\begin{tabular}{|c|c|c|c|c|c|c|c|c|c|}
\hline \multirow{2}{*}{ Exp. } & \multirow{2}{*}{ Femelles } & \multirow{2}{*}{ Mâles } & \multirow{2}{*}{$\begin{array}{c}\text { Rétention } \\
\text { totale }\end{array}$} & \multirow{2}{*}{$\begin{array}{l}\text { Oeufs } \\
\text { déposés } \\
\text { (total) }\end{array}$} & \multirow{2}{*}{$\begin{array}{c}\% \\
\text { Survie }\end{array}$} & \multicolumn{3}{|c|}{ Descendance $(\%)$} & \multirow{2}{*}{$\begin{array}{c}\text { Effectif } \\
\text { échantillonné }\end{array}$} \\
\hline & & & & & & Saumon & Hybride & Truite & \\
\hline 1 & $1 \mathrm{~S}$ & $1 S+1 \operatorname{tac}$ & 210 & 4487 & 37.2 & 100 & - & - & 93 \\
\hline 2 & $1 \mathrm{~S}$ & $1 S+6$ tac & 1 & 4478 & 41.8 & 100 & - & - & 77 \\
\hline 3 & $1 S$ & 6 tac & 0 & 4225 & 16.1 & 100 & - & - & 100 \\
\hline 4 & $1 \mathrm{~S}$ & $1 S+6 T$ & 183 & 3705 & 55.2 & 100 & 0 & - & 100 \\
\hline 5 & $1 S$ & $1 S+6 T$ & 0 & 4352 & 13.6 & 100 & 0 & - & 100 \\
\hline 6 & $1 \mathrm{~S}$ & $3 T$ & - & 4237 & 26.6 & - & 100 & - & 100 \\
\hline 7 & $1 \mathrm{~S}$ & $4 T+3 \operatorname{tac}$ & 1211 & 3113 & 42.2 & 21 & 79 & - & 100 \\
\hline 8 & $1 S$ & $3 T+6$ tac & 0 & 4306 & 15.5 & 100 & 0 & 0 & 100 \\
\hline 9 & $5 T$ & $6 \mathrm{~T}$ & 65 & 650 & 59.7 & - & - & 100 & 100 \\
\hline 10 & $5 T$ & $6 T+6$ tac & 76 & 1007 & 40.2 & - & 0 & 100 & 100 \\
\hline 11 & $5 \mathrm{~T}$ & $6 T+6 \operatorname{tac}$ & 54 & 849 & 70.6 & - & 0 & 100 & 100 \\
\hline 12 & $5 \mathrm{~T}$ & $6 \operatorname{tac}$ & 941 & 182 & 0 & - & $100^{*}$ & - & $11^{*}$ \\
\hline 13 & $5 \mathrm{~T}$ & 6 tac & 459 & 1505 & 1.3 & $\cdot$ & 100 & - & 18 \\
\hline 14 & $5 \mathrm{~T}$ & $1 \mathrm{~S}+6 \mathrm{tac}$ & 499 & 924 & 1.95 & - & 100 & - & 9 \\
\hline
\end{tabular}

* oeufs seulement

Les analyses génétiques n'indiquent la présence d'hybrides que dans les cas de sympatrie où les mâles appartenant à la même espèce que la femelle étaient absents (Tabl. II : exp. $6,12,13,14)$, avec une seule exception, l'expérience 7 . Dans ce dernier cas, 4 truites de 16 à $34 \mathrm{~cm}$ et 3 tacons de 11 à $14 \mathrm{~cm}$ étaient présents avec la femelle saumon, et $79 \%$ des descendants sont des hybrides. Dans le cas de l'expérience 8 , dont les conditions sont semblables à celles de l'expérience 7 , les analyses génétiques n'indiquent pas de présence d'hybrides. Aucun hybride n'est trouvé dans les situations où soit le mâle saumon avec la femelle saumon (exp. 4 et 5) soit les mâles truites avec les femelles truites (exp. 10 et 11) sont en compétition avec des mâles hétérospécifiques. Par ailleurs, les tacons seuls sont parfaitement capables de se reproduire avec une femelle saumon (exp. 3), de même qu'ils peuvent frayer avec des femelles truites (exp. 12, 13, 14). 


\subsubsection{Rétention et succès reproducteur}

Le nombre total d'oeufs déposés dépend fortement de la rétention d'ovules des femelles (Tabl. II). Dans des expériences conduites en dehors de cette étude, la rétention est en moyenne de 141 ovules $(3,4 \%)$ par femelle pour 10 femelles saumons associées séparément à 10 mâles (BEALL, 1996). Pour les saumons de la présente étude, la rétention varie de 0 à $28 \%$. Elle est faible ( $(0$ et $4,5 \%$ ) dans les biefs témoins (expériences 1 et 2 ). En présence des tacons seuls (exp. $3)$, il n'y a pas de rétention, et la présence des truites à côté du mâle saumon ne semble pas gêner la femelle saumon (exp. 4 et 5). La rétention est la plus forte dans l'expérience 7 (28\%), où il n'y a pas de saumon mâle anadrome. Chez les femelles truites, la rétention est de $9,1 \%$ dans le témoin (exp. 9) et comparable dans les biefs où les mâles sont sympatriques ( 7 et $6 \%$, respectivement, dans les exp. 10 et 11). Par contre, la rétention des femelles truites est très élevée ( 23 à $84 \%$ ) dans les trois dernières expériences où il n'y a que des mâles saumons.

\subsubsection{Survie}

De même, la survie varie fortement selon les conditions, de 13,6 à 55,2\% pour la progéniture des femelles saumons, et de 0 à $70,6 \%$ pour celle des truites (Tabl. II). Dans les biefs témoin saumon (exp. 1 et 2), elle est proche de $40 \%$. Une faible survie $(16,1 \%)$ est enregistrée dans l'expérience 3 avec les tacons mâles. Le même résultat est obtenu dans l'expérience 8 , où malgré la présence des truites mâles, tous les oeufs sont fécondés par les tacons mâles précoces, mais avec une faible efficacité (15,5\% de survie). Une grande variabilité, difficile à expliquer, est trouvée dans les expériences 4 et 5 . Pour les truites femelles, la survie est bonne dans les trois biefs avec les mâles truites, avec ou sans tacons (exp. 9, 10, $11: 40$ à $71 \%$ ). Par contre, la survie des hybrides dans les trois dernières expériences est très faible, 0 à $2 \%$. Dans l'expérience 12, seuls quelques oeufs vivants avaient été déterrés du gravier, mais aucun alevin n'a survécu.

\subsubsection{Durée de l'activité de frai et nombre de frayères}

Nous avons pris comme référence des données obtenues sur 10 couples de saumon isolés, en dehors de cette étude, dans des conditions normales de reproduction où la femelle est mise en place au stade de l'ovulation (BEALL, 1996). L'activité de frai de ces femelles commence par des creusements 1,1 jour après la mise en place et dure 4,3 jours. Une femelle en présence d'un mâle saumon et de truites mâles démarre dans les mêmes conditions mais reste active plus longtemps (6 jours, exp. 4 et 5 ). Avec des tacons mâles seuls (exp. 3 ), elle met aussi 6 jours pour pondre tous ses oeufs. Lorsque ce sont des truites mâles, elle met 8 jours (exp. 6), et lorsque les mâles sont des truites et des tacons, l'activité totale dure 9 jours (exp. 7 et 8 ). Dans ce dernier cas, elle démarre beaucoup plus tard, en moyenne 8 jours après la mise en place dans le chenal. Sept des huit femelles saumons creusent une frayère, la femelle de l'expérience 4 en forme deux.

Chez les truites femelles, on constate une différence entre les expériences 9, 10, 11 (mâles truites, seuls ou avec tacons), avec une durée moyenne de 3,7 jours pour le démarrage et de 13 jours d'activité totale, et les expériences $12,13,14$, où les mâles sont des saumons (démarrage 6,7 jours et durée totale 14,3 jours). Lorsque les mâles truites sont présents, avec ou sans tacons, le nombre de frayères est homogène $(2 /$ femelle) et la surface moyenne par femelle se situe entre 0,39 et $0,61 \mathrm{~m}^{2}$. En présence des mâles saumons seuls, les résultats sont beaucoup plus variables, en liaison avec le fait que certaines femelles retiennent tout ou partie de leurs oeufs. Ainsi, dans l'expérience 12, une seule truite dépose des oeufs, quatre frayent dans l'exp. 13 et trois dans la 14. Le nombre moyen de frayères/femelle varie ainsi de 0.6 à 3 , et la surface de 0.086 à $0.58 \mathrm{~m}$ ?.

\subsubsection{Analyse comportementale}

Les fréquences des principaux comportements de reproduction considérés comme pertinents dans cette étude (pour la femelle : creusements, sorties de la frayère et chasses ou menaces envers le ou les mâle(s); pour le mâle dominant qui courtise la femelle : vibrations, sorties et chasses vis-à-vis des autres mâles) sont présentées figure 1, à titre d'exemple pour certaines situations. Plus de détails sont donnés dans BEALL (1996). 


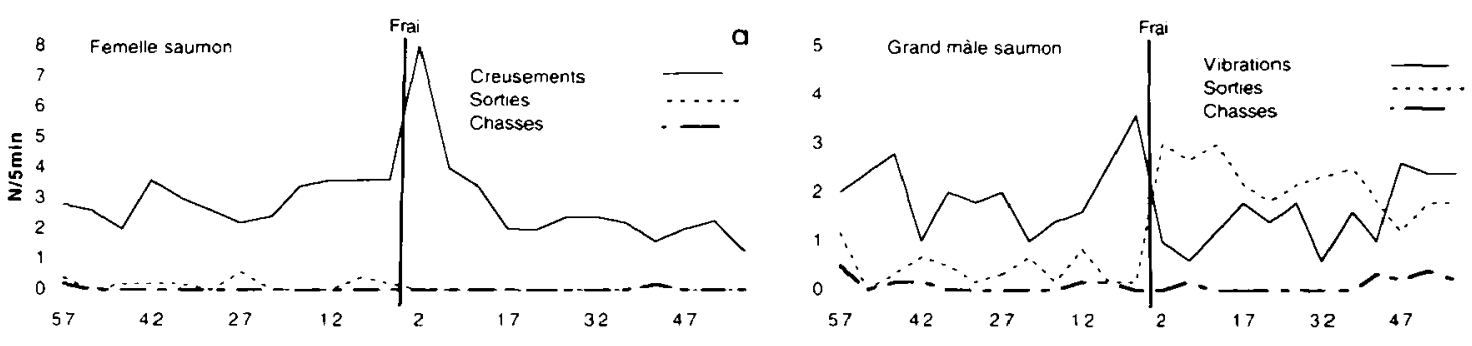

b
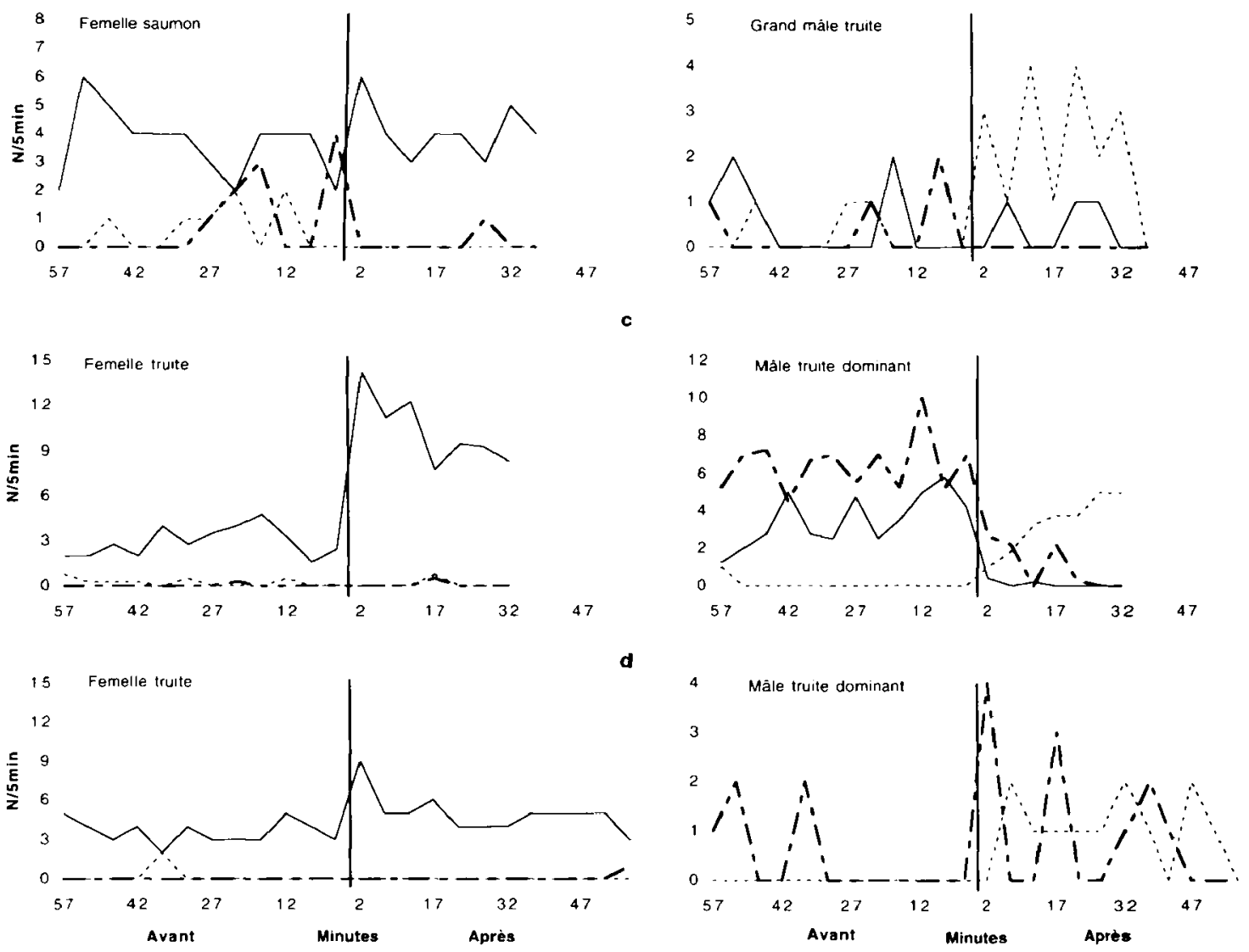

Figure 1

Fréquences des comportements, par périodes de 5 minutes, pendant $1 \mathrm{~h}$ avant et $1 \mathrm{~h}$ après le frai, des femelles et des mâles dominants des deux espèces dans différentes situations.

a : couple de saumons avec tacons (exp. 2 ; moyenne de 5 séquences d'observation) ;

b : femelle saumon et mâles truites (exp. 6 ; 1 séquence d'observation) ;

c : truites du ruisseau de Muniellos (moyenne de 5 séquences);

d : truites femelles et mâles avec tacons (exp. 11 ; 1 séquence d'observation).

\section{Figure 1}

Frequencies over $5 \mathrm{~min}$. intervals of main behaviours during the 1-h periods preceding and following spawning, for females and dominant males of the two species in different situations.
a : salmon pair with parrs (exp. 2 ; mean of 5 observation sequences) ;
b : female salmon and male trouts (exp. 6 ; 1 sequence) ;
$c$ : trouts in the Muniellos brook (mean of 5 observation sequences) ;
d : female and male trouts with parrs (exp. 11 ; 1 sequence). 
Dans les conditions normales de reproduction (Fig. 1a, exp. 2), la femelle saumon creuse régulièrement au rythme de 2 à 4 séries par 5 min. jusqu'au moment du frai, redouble d'activité pendant une dizaine de minutes juste après le frai (recouvrement des oeufs), puis revient à un rythme de 2 creusements par 5 min. Elle sort rarement de la zone de frayère et ne montre aucun comportement agressif envers le mâle qui la courtise, ni les tacons. Le mâle est également très actif dans la période avant le frai, avec une fréquence élevée du comportement de cour le plus typique, la vibration. Les sorties de la frayère sont relativement fréquentes avant le frai, mais elles sont associées à des chasses sur les tacons en dehors de la zone de frayère et un retour rapide auprès de la femelle. Par contre, une fois le frai accompli, le mâle tend à s'éloigner plus souvent et pendant des intervalles plus longs.

En présence de truites mâles (Fig.1b, exp. 6), la femelle quitte souvent la frayère et chasse les truites mâles. L'activité du mâle truite dominant est complètement atypique (Fig.1b) car il courtise peu la femelle saumon.

Chez les truites sédentaires, qui servent de référence (ruisseau de Muniellos, Fig. 1c), les femelles ont une activité de creusement avant le frai régulière et comparable à celle des saumons. Cette activité augmente très fortement après le frai, passant de 3 séries de creusements / $5 \mathrm{~min}$. à plus de 12 et même $15 / 5 \mathrm{~min}$. Les sorties de la frayère sont inexistantes au cours de la période d'observation, de même que les chasses avant le frai. Le mâle dominant (Fig. 1c), avant le frai, courtise très activement la femelle, reste en permanence à ses côtés, et la défend avec acharnement par des chasses sur les autres truites mâles. Après le frai, il ne courtise plus la femelle, sort fréquemment et chasse moins les autres truites.

Dans l'expérience 11 (Fig. 1d), la femelle truite a une activité comparable à celle des truites de référence avant le frai, mais plus faible après. Le mâle truite dominant courtise peu la femelle, mais il chasse activement les autres truites mâles et les tacons.

Ces figures montrent que les femelles poursuivent leur activité de frai (creusement), avec quelques modifications en présence des mâles de l'autre espèce. Le mâle saumon attaque peu souvent les tacons, mais lorsqu'il le fait les chasses sont violentes. Les mâles truites, en présence de compétiteurs truites ou tacons, sont très agressifs. Ces résultats sont globalement confirmés par les tests de KRUSKAL-WALLIS, effectués sur l'ensemble des situations (BEALL, 1996).

\section{4 - DISCUSSION ET CONCLUSION}

Le taux d'hybridation de $2,8 \%$ trouvé dans cinq rivières des Asturies est tout à fait comparable à celui cité par GARCIA DE LEANIZ et VERSPOOR (1989), pour des rivières voisines de la province de Cantabria, et reste du même ordre de grandeur pour une rivière du Pays Basque analysée dans le sud-ouest de la France. Le phénomène semble donc bien répandu à l'échelle du continent européen, avec des taux moyens assez faibles mais pouvant atteindre localement des valeurs élevées, comme $9,4 \%$ sur une station de la Narcea, $28 \%$ dans un échantillon de la rivière Grönan en Suède (JANSSON et al., 1991), et $31 \%$ sur un affluent de la Baltique (SEMYONOVA et SLYN'KO, 1988). Les fortes variations constatées d'un prélèvement ou d'une station à l'autre peuvent être simplement dues à un échantillonnage peu représentatif, mais également traduire une situation locale particulière ou déséquilibrée.

En effet, d'après les expériences réalisées dans les conditions naturelles du chenal de frai, en situation normale de reproduction, avec des géniteurs des deux sexes en sympatrie, les mécanismes comportementaux d'isolement reproducteur sont très forts. Ainsi, dans la période précédant le frai le mâle dominant de l'une ou l'autre espèce, qui déjà chasse les mâles compétiteurs de sa propre espèce (BEALL, 1994), attaque vigoureusement les mâles hétérospécifiques. Les mâles représentent donc la principale barrière pré-appariement. Par ailleurs, différentes études menées actuellement dans le chenal de frai du Lapitxuri (BONZOM et BEALL, 1995 ; DE GAUDEMAR, comm. pers.) indiquent que les femelles saumons préfèrent s'apparier avec des mâles aussi ou plus grands qu'elles. 
En l'absence de mâles anadromes conspécifiques, la femelle saumon serait moins sélective. En effet, elle accepte de se reproduire avec des tacons mâles précoces seuls (expérience 3 ; MYERS et HUTCHINGS, 1987), qui sont pourtant plus de 100 fois plus petits qu'elle et n'offrent pas de stimuli et de comportement de cour comparables à ceux des grands mâles anadromes. Mais elle accepte également la présence des mâles truites (exp. 7). II est possible que pour elle les petits mâles truites n'apparaissent pas différents des tacons. Une autre possibilité serait que les petites truites adoptent la tactique de reproduction du " sneaker " caractéristique des tacons. Dans l'un ou l'autre cas, l'hypothèse semble renforcée par le fait que ce n'est pas la plus grosse truite $(34 \mathrm{~cm})$ qui est à l'origine des hybrides (d'après des analyses de paternité effectuées dans ce bief), mais un mâle de taille intermédiaire, un peu plus gros qu'un tacon. En effet, la truite de $34 \mathrm{~cm}$ a moins de chance de passer inaperçue et de leurrer la femelle saumon que les poissons de 12 à $22 \mathrm{~cm}$.

Cependant, même si la femelle accepte de se reproduire avec des mâles hétérospécifiques, elle modifie son comportement conformément à la théorie de la sélection sexuelle. Ainsi, elle tend à allonger la période d'activité par rapport aux témoins dès lors que les mâles de l'autre espèce sont présents, peut-être en raison du temps passé à les chasser. En l'absence du mâle saumon, le début du frai est retardé et l'activité dure plus longtemps. De même, une femelle saumon non perturbée par la présence d'individus mâles étrangers tend à construire rapidement une frayère homogène, alors qu'une femelle sans mâle anadrome se disperse dans ses creusements et augmente la durée de cette activité (BEALL, 1996). Chez les truites femelles, le phénomène est plus prononcé et se traduit par des rétentions plus élevées, dues au fait que certaines femelles ne se reproduisent pas du tout. Une rétention élevée est généralement indicatrice d'une activité de frai perturbée (BEALL, 1994). Ces modifications comportementales, tendant à retarder le moment des pontes et à étaler la durée de la reproduction, pourraient être une adaptation permettant à des mâles conspécifiques d'arriver et de supplanter les mâles hétérospécifiques.

Dans les conditions normales de reproduction dans le chenal (faibles densités de géniteurs, conditions environnementales favorables), la survie est de l'ordre de $40 \%$ chez le saumon (BEALL et al., 1991) et de 40 à $70 \%$ pour la truite (cette étude). Avec des tacons mâles précoces seuls, la survie des descendants d'une femelle saumon tombe à $16 \%$. Cette faible survie peut être causée par une moindre efficacité des tacons qui, en raison de leur petite taille, épuisent rapidement leurs gonades par rapport à celles des grands mâles. Comme une femelle pond tous ses oeufs en une dizaine de fois sur une courte période (BEALL, 1994), ceux qui participent aux premiers frais sont moins efficaces par la suite (THOMAZ, 1995). La survie d'hybrides issus du croisement femelle saumon x mâle truite est de l'ordre de $25 \%$ (exp. 6). Dans le cas des hybrides issus de femelles truites, les mortalités sont très importantes, entre 98 et $100 \%$. MCGOWAN et DAVIDSON (1992 b) observent le même type de mortalité différentielle, dans des conditions d'élevage. II pourrait donc exister une deuxième barrière d'isolement reproducteur de type post-appariement, peut-être plus prononcée chez la truite. Ceci pourrait être dû à la taille plus petite des oeufs de truite, qui ne laisserait pas suffisamment de place à l'embryon hybride pour se développer normalement (MCGOWAN et DAVIDSON, 1992 b). Cependant, CHEVASSUS (1979) rend compte de viabilités comparables pour les deux types de $F 1$, les différences observées pouvant tenir à la taille des femelles de truite commune (truite de mer ou truite sédentaire). Dans nos expériences, les femelles truites étaient toutes de petite taille.

AVISE et SAUNDERS (1984) émettent l'hypothèse que l'espèce la plus rare dans un croisement hybride tend à fournir le parent femelle, l'absence de partenaire conspécifique et de stimuli de cour pour la femelle de l'espèce rare pouvant être des facteurs plus importants dans la probabilité d'augmentation de l'hybridation interspécifique.

Dans nos régions, l'espèce plus rare est généralement le saumon, avec souvent quelques centaines, voire dizaines de géniteurs arrivant jusqu'aux zones de frayères. Nous avons suivi en décembre 95 et janvier 96 l'évolution des populations de géniteurs de saumon, truite de mer et truite sédentaire sur certaines frayères du Gave d'Oloron. En début de période, 
le sexe ratio semblait équilibré, alors qu'en fin de saison (mi-janvier), il ne restait pratiquement plus de gros mâles ni de castillons. Ce déséquilibre pourrait être causé par des mortalités différentielles subies par les mâles, en raison des dépenses énergétiques, et des blessures ouvrant la voie aux agents pathogènes, encourues lors de la recherche des femelles actives et des combats incessants livrés contre les autres mâles pour défendre l'accès à ces femelles (BEALL, 1994). Quelques femelles saumons tardives poursuivaient leur activité, et elles étaient alors courtisées activement par des mâles truites, et entourées de tacons. Les conditions permettant l'hybridation étaient alors réunies. II faut noter ici que les tacons mâles précoces, qui peuvent représenter plus de 100 fois la population de géniteurs anadromes (L'ABEE-LUND, 1989), pourraient servir de réservoir de mâles pour les femelles saumons, selon l'hypothèse de JONES (1959), en prenant le relais des mâles défaillants. Ils pourraient également, par leur seule présence et leur nombre, minimiser l'incidence de l'hybridation par les truites mâles.

Le fait que des mortalités différentielles très fortes existent pour les hybrides issus de femelles truites, et que l'on ne trouve pas ce type d'hybride dans les rivières européennes, indique que s'il y a hybridation dans le sens femelle truite $x$ mâle saumon, le potentiel reproducteur de la truite est gaspillé. Ceci pourrait être le cas au Canada, où la truite est une espèce introduite depuis peu, et donc la plus rare, mais les populations ne semblent pas péricliter.

Les résultats obtenus au cours de cette étude montrent que l'impact des repeuplements sur l'incidence de l'hybridation est, apparemment, minimal. En effet, les analyses génétiques montrent que les pères des hybrides sont tous des truites sauvages. Par ailleurs, les analyses de I'ADN mitochondrial indiquent que dans nos régions les hybrides sont issus de femelles saumons, et donc que les tacons ne jouent aucun rôle dans cette hybridation. Cependant, en raison des mortalités différentielles constatées expérimentalement dans le chenal de frai et en laboratoire, il est possible que des hybrides femelle truite $x$ mâle saumon existent, comme c'est le cas au Canada, mais ne survivent pas suffisamment longtemps, ou en assez grand nombre, pour être échantillonnés. Les études doivent être poursuivies pour évaluer l'ampleur du détournement de potentiel reproducteur de la truite, s'il existe.

La détermination du sens de croisement par l'ADN mitochondrial simplifie les possibilités concernant le test des hypothèses de départ (rôle des sujets de repeuplements et plus particulièrement des tacons mâles précoces). Les truites mâles de repeuplement ne jouent aucun rôle et les tacons mâles précoces ne semblent pas impliqués. Par contre, l'éventuelle participation de femelles saumons issues d'élevage n'a pu être déterminée en raison du manque de marqueurs génétiques capables de discriminer entre les sujets sauvages et de repeuplement. Cependant, grâce à la teneur en certains pigments caroténoïdes, une méthode non génétique, mise au point par YOUNGSON et al. (1993), a mis en évidence le rôle majeur des saumons femelles échappés des cages marines d'élevage dans l'incidence de l'hybridation en Écosse.

En conséquence, il est recommandé de limiter les actions anthropiques susceptibles d'augmenter ou de favoriser l'incidence de l'hybridation. Les aires de reproduction, dont la réduction favorise la promiscuité, doivent être rendues facilement accessibles, particulièrement aux migrateurs, protégées et éventuellement restaurées ou améliorées. La structure des populations de géniteurs, avec toutes ses composantes, doit être maintenue en équilibre, avec des effectifs suffisants de géniteurs et en particulier de mâles des deux espèces. L'introduction d'une espèce non indigène dans l'aire de distribution d'une espèce proche phylogénétiquement doit être évitée (exemple, parmi d'autres, de la truite commune introduite au Canada). Enfin, il convient de limiter les repeuplements avec des sujets non autochtones, particulièrement dans le cas des saumons juvéniles, tant que leur rôle n'aura pas été précisément évalué.

\section{5 - REMERCIEMENTS}

Cette étude a été entreprise dans le cadre d'une action intégrée franco-espagnole Picasso en 1993 et 1994 ( $n^{\circ} 93187$ côté français et DGICYT HF 55B et 232B côté espagnol), et 
avec le soutien d'un contrat INRA-Région Aquitaine $n^{\circ} 940308004$ pour 1994. Nous tenons à remercier l'APPMA de la Nivelle et son président, J. CHOUFFOT, pour l'aide apportée dans les collectes de poissons. La Délégation Régionale du CSP de Pau a fourni les échantillons de la Nive. Ils ont été analysés par L. BOURKE, de I'Université de Cork (Irlande), qui nous a gracieusement fait part de ses résultats.

\section{6 - BIBLIOGRAPHIE}

AVISE J.C., SAUNDERS N.C., 1984. Hybridization and introgression among species of sunfish (Lepomis) : analysis by mitochondrial DNA and allozyme markers. Genetics, 108, 237255.

BEALL E., 1994. Les phases de la reproduction. In GUEGUEN J.C., PROUZET P., eds., Le saumon atlantique, 123-140, IFREMER, Plouzané.

BEALL E., 1996. Mécanismes de l'hybridation entre la truite commune et le saumon atlantique. Rapport contrat INRA-Région Aquitaine, $n^{\circ}$ 940308004, INRA, Station d'Hydrobiologie de St-Pée-sur-Nivelle, $45 \mathrm{p}$.

BEALL E., MARTY C., 1983. Reproduction du saumon atlantique, Salmo salar L., en milieu semi-naturel contrôlé. Bull. Fr. Piscic., 289, 77-93.

BEALL E., MARTY C., HELAND M., 1991. Production de juvéniles de salmonidés pour le repeuplement, chenal de frai du Lapitxuri : bilan 1981-1988. Convention d'étude Etat/INRA $\mathrm{n}^{\circ} 2651$ B, Etude des conditions optimales de développement des populations de saimonidés migrateurs, INRA, Station d'Hydrobiologie de St-Pée-sur-Nivelle, 56 p.

BELAND K.F., ROBERTS F.L., SAUNDERS R.L., 1981. Evidence of Salmo salar x Salmo trutta hybridization in a North American river. Can. J. Fish Aquat. Sci., 38, 552-554.

BONZOM J.M., BEALL E., 1995. Modification du comportement de reproduction de la femelle saumon (Salmo salar) en fonction de la taille du mâle. In NUNEZ DE MURGA J., NUNEZ DE MURGA M., GARCIA BRULL P.D., MORALES L.O., eds., Motivacion, 149-161, Universidad de Valencia, Grafiques Machi, Valencia.

CAMPTON D.E., 1987. Natural hybridization and introgression in fishes. Methods of detection and genetic interpretations. In RYMAN N., UTTER F., eds., Population Genetics and Fishery Management, 161-192, University of Washington Press, Seattle.

CHEVASSUS B., 1979. Hybridization in salmonids : results and perspectives. Aquaculture, 17, 113-128.

CROZIER W.W., 1984. Electrophoretic identification and comparative examination of naturally occurring $\mathrm{F} 1$ hybrids between brown trout (Salmo trutta L.) and Atlantic salmon (Salmo salar L.). Comp. Biochem. Physiol., 78B, 785-790.

CROZIER W.W., 1993. Evidence of genetic interaction between escaped farmed salmon and wild Atlantic salmon (Salmo salar L.) in a Northern Irish river. Aquaculture, 113, 19-29.

GARCIA DE LEANIZ C., VERSPOOR E., 1989. Natural hybridization between Atlantic salmon, Salmo salar, and brown trout, Salmo trutta, in northern Spain. J. Fish Biol., 34, 41-46.

GUYOMARD R., 1978. Identification par électrophorèse d'hybrides de Salmonidés. Ann. Genet. Sél. Anim., 10, 17-27.

HEGgBeRget T.G., HAUKEBO T., MORK J., STAHL G., 1988. Temporal and spatial segregation of spawning in sympatric populations of Atlantic salmon, Salmo salar L., and brown trout, Salmo trutta L. J. Fish Biol., 33, 347-356.

HELAND M., DUMAS J., 1994. Ecologie et comportement des juvéniles. In GUEGUEN J.C., PROUZET P., eds., Le saumon atlantique, 29-46, IFREMER, Plouzané. 
HINDAR K., RYMAN N., UTTER F., 1991. Genetic effects of cultured fish on natural fish populations. Can. J. Fish. Aquat. Sci., 48, 945-957.

HURREL R.H., PRICE D.J., 1991. Natural hybrids between Atlantic salmon, Salmo salar L., and trout, Salmo trutta L., in juvenile salmonid populations in south-west England. J. Fish Biol., 39A, 335-341.

JANSSON H., HOLMGREN I., WEDIN K., ANDERSSON T., 1991. High frequency of natural hybrids between Atlantic salmon, Salmo salar L., and brown trout, S. trutta L., in a Swedish river. J. Fish Biol., 39A, 343-348.

JOHNSON M.S., WRIGHT J.E., 1986. Female brown trout $\times$ Atlantic salmon hybrids produce gynogens and triploids when backcrossed to male Atlantic salmon. Aquaculture, 57, 345-358.

JONES J.W., 1959. The salmon. Harper and Bros, New York, $192 \mathrm{p}$.

KRIEG F., GUYOMARD R., 1985. Population genetics of French brown trout (Salmo trutta L.) : large geographical differentiation of wild populations and high similarity of domesticated stocks. Génét. Sél. Evol., 17, 225-242.

L'ABEE-LUND J.H., 1989. Significance of mature male parr in a small population of Atlantic salmon (Salmo salar). Can. J. Fish. Aquat. Sci., 46, 928-931.

MCGOWAN C., DAVIDSON W.S., 1992 a. Unidirectional natural hybridization between brown trout (Salmo trutta) and Atlantic salmon (S. salar) in Newfoundland. Can. J. Fish. Aquat. Sci., 49, 1953-1958.

MCGOWAN C., DAVIDSON W.S., 1992 b. Artificial hybridization of Newfoundland brown trout and Atlantic salmon : hatchability, survival and growth to first feeding. Aquaculture, 106, 117-125.

MORAN P., PENDAS A.M., GARCIA-VAZQUEZ E., IZQUIERDO J.I., 1991. Failure of a stocking policy, of hatchery reared brown trout, Salmo trutta L., in Asturias, Spain, detected using $\mathrm{LDH}-5^{\star}$ as a genetic marker. J. Fish Biol., 39A, 117-121.

MYERS R.A., HUTCHINGS J.A., 1987. Mating of anadromous Atlantic salmon (Salmo salar L.) with mature male parr. J. Fish Biol., 31, 143-146.

NICIEZA A.G., BRANA F., 1995. Estructura de la poblacion reproductora, edad de maduracion y principales parametros reproductivos. In BRANA F., ed., Biologia y conservacion del salmon atlantico (Salmo salar) en los rios de la region cantabrica, 147-160, ICONA, Madrid.

NYMAN L., 1970. Electrophoretic analysis of hybrids between salmon (Salmo salar L.) and trout (Salmo trutta L.). Trans. Am. Fish. Soc., 99, 229-236.

PAYNE R.H., CHILD A.R., FORREST A., 1972. The existence of natural hybrids between the European trout and the Atlantic salmon. J. Fish Biol., 4, 233-236.

SEMYONOVA S.K., SLYN'KO V.I., 1988. Polymorphism of proteins in populations of Atlantic salmon (Salmo salar L.), brown trout (S. trutta L.) and their hybrids. Genetika USSR, 24, 548-555.

SOKAL R.R., ROHLF F.J., 1969. Biometry. Freeman W.H. and Co., San Francisco, 776 p.

STEARLEY R.F., 1990. Historical biology of Oncorhynchus. PhD Thesis, University of Michigan, $158 \mathrm{p}$.

TAGGART J.B., HYNES R.A., PRODOHL P.A., FERGUSON A., 1992. A simplified protocol for routine total DNA isolation from salmonid fishes. J. Fish Biol., 40, 963-965.

THOMAZ D., 1995. Alternative life-history strategies in male Atlantic salmon (Salmo salar L.). PhD Thesis, University of Leicester, $132 \mathrm{p}$. 
VERSPOOR E., 1988. Widespread hybridization between Atlantic salmon, Salmo salar, and introduced brown trout, S. trutta, in eastern Newfoundland. J. Fish Biol., 32, 327-334.

YOUNGSON A.F., KNOX D., JOHNSTONE R., 1992. Wild adult hybrids of Salmo salar L. and Salmo trutta L. J. Fish Biol., 40, 817-820.

YOUNGSON A.F., WEBB J.H., THOMPSON C.E., KNOX D., 1993. Spawning of escaped farmed Atlantic salmon (Salmo salar) : hybridization of females with brown trout (Salmo trutta). Can. J. Fish. Aquat. Sci., 50, 1986-1990.

ZIMMERMAN E.G., AKINS D.R., PLANZ J.V., SCHURR M.J., 1988. A rapid procedure for isolating mitochondrial DNA. Gene. Anal. Techn., 5, 102-104. 